\section{Case Reports in Ophthalmology}

Case Rep Ophthalmol 2021;12:11-15

DOI: $10.1159 / 000511100$

Published online: January 7, 2021 (c) 2021 The Author(s)

Published by S. Karger AG, Basel www.karger.com/cop

\title{
Nonarteritic Anterior Ischemic Optic Neuropathy following Herpes Zoster Ophthalmicus and Steroid-Related Intraocular Pressure Spike
}

\author{
Po Hsiang Yuan ${ }^{a}$ Jonathan A. Micieli ${ }^{b-d}$ \\ aFaculty of Medicine, University of British Columbia, Vancouver, BC, Canada; ${ }^{b}$ Department \\ of Ophthalmology and Vision Sciences, University of Toronto, Toronto, ON, Canada; \\ 'Division of Neurology, Department of Medicine, University of Toronto, \\ Toronto, ON, Canada; ${ }^{d}$ Kensington Vision and Research Centre, Toronto, ON, Canada
}

\section{Keywords}

NAION · Ischemic optic neuropathy · Intraocular pressure $\cdot$ Herpes zoster

\begin{abstract}
A 61-year-old woman presented with sudden vision loss from a left anterior optic neuropathy in the context of severely elevated intraocular pressure after starting topical steroids for anterior uveitis related to herpes zoster ophthalmicus (HZO). The strong temporal relationship between the vision loss and elevated IOP suggested the vision loss was related to nonarteritic anterior ischemic optic neuropathy (NAION). Anterior chamber paracentesis did not detect varicella zoster virus (VZV) and magnetic resonance imaging of the orbits was normal. Her vision remained stable and the optic disc edema resolved within 3 months. The occurrence of NAION following an acute elevation of intraocular pressure (IOP) is well-documented in the context of surgical procedures, glaucoma, uveitis and trauma. This case indicates that not every optic neuropathy in close temporal relationship with $\mathrm{HZO}$ is directly attributable to the virus. NAION may also occur after steroid-related IOP spikes and ophthalmologists should ensure that patients on topical steroids are closely monitored for ocular complications.
\end{abstract}




\section{Case Reports in Ophthalmology}

\section{Introduction}

Vision loss is a rare complication of herpes zoster ophthalmicus (HZO) and can be a result of anterior segment of posterior segment involvement. Optic neuropathy is a rare complication of $\mathrm{HZO}$ seen in less than $0.5 \%$ of cases and can be anterior (associated with optic disc edema) or retrobulbar [1]. The diagnosis of herpes zoster optic neuropathy (HZON) is diagnosed in patients who develop an optic neuropathy in close temporal relationship with HZO with other causes of optic neuropathy being excluded. Supportive evidence for the diagnosis is helpful by means of magnetic resonance imaging (MRI) of the orbits with contrast demonstrating enhancement of the optic nerve in retrobulbar cases, or the detection of varicella zoster virus (VZV) in the aqueous humor or cerebrospinal fluid (CSF) [1]. However, the yield of detection of VZV in the CSF is low, especially in anterior cases. Treatment consists of oral or intravenous antivirals and the use of systemic corticosteroids remains controversial [1]. Patients with HZO may develop an anterior optic neuropathy that is not directly related to VZV, but secondarily related to HZO by means of other ocular complications. We report a case of nonarteritic anterior ischemic optic neuropathy (NAION) related to HZO, which was likely secondary to the development of a steroid response and elevated intraocular pressure (IOP) for the treatment of anterior uveitis. This indicates that not every optic neuropathy in close temporal relationship to $\mathrm{HZO}$ is directly related to the virus.

\section{Case Report}

A 61-year-old woman was seen in neuro-ophthalmology consultation for a left optic neuropathy. She had a history of hypertension and was taking amlodipine. She developed left HZO 1 month prior to presentation with skin lesions on the left forehead and tip and side of her nose. She was treated with valacyclovir $1 \mathrm{~g}$ PO TID for 10 days. She saw an ophthalmologist 3 days later and was found to have anterior uveitis (1+ cells), an intraocular pressure of 18 , and was started on prednisolone acetate $1 \%$ eye drops QID. This was continued and the examination remained stable 1 week later. Two weeks later, she developed left eye pain and worsening vision. She saw an ophthalmologist and was found to have an intraocular pressure of $52 \mathrm{~mm}$ $\mathrm{Hg}$ without any signs of uveitis. Her refraction was $-1.00+0.50 \times 172$ OD and $-1.50+0.50 \times$ $180 \mathrm{OS}$ and the gonioscopy revealed open angles, grade 3 (Shaffer system) with the trabecular meshwork visible for 360 degrees in both eyes. She was started on acetazolamide $250 \mathrm{mg}$ PO QID, brimonidine-timolol eye drops BID and topical prednisolone acetate was discontinued given the likelihood of a steroid response. Follow-up the next day revealed an improved intraocular pressure of 16 , but she was noted to have a new relative afferent pupillary defect and optic disc edema in the left optic nerve. A neuro-ophthalmology consultation was requested. Acetazolamide and brimonidine-timolol eye drops were discontinued.

Neuro-ophthalmology assessment revealed a visual acuity of 20/20 OD and 20/100 OS, a left RAPD, and Humphrey visual field testing demonstrated a left inferior altitudinal visual field defect (Fig. 1a). Anterior segment revealed no signs of uveitis and dilated fundus examination demonstrated a normal appearing right optic nerve with a cup-to-disc ratio of 0.1 and left diffuse optic disc edema. Optical coherence tomography of the retinal nerve fiber layer showed an average thickness of $84 \mu \mathrm{m}$ OD and $182 \mu \mathrm{m}$ OS (Fig. 1b). The differential diagnosis considered was a left anterior optic neuropathy related to herpes zoster or NAION related to the recent IOP elevation. An anterior chamber paracentesis was performed and VZV was not detected by real-time PCR. She had an MRI of the brain and orbits with contrast that did not 
show enhancement of the optic nerves and was normal. Complete blood count, erythrocyte sedimentation rate and C-reactive protein were normal. She was treated with valacyclovir $1 \mathrm{~g}$ PO TID for 1 month as a precaution, but her vision did not improve. At 3-month follow-up, she continued to have a left inferior altitudinal visual field defect and the left optic disc edema had resolved.

\section{Discussion}

Vision loss is a feared complication of HZON and may affect almost any part of the eye including the anterior and posterior segments [1]. This may be a result of direct involvement of VZV or a secondary inflammatory component. This case highlights that optic nerve complications from VZV may not be due to direct involvement of the virus but can be a secondary result of another ocular complication. Our patient developed NAION related to elevated IOP that likely disrupted the perfusion to the optic nerve head. This was unlikely to be a direct result of the virus for a number of reasons including the absence of detectable virus in the aqueous humor by PCR, the absence of any optic nerve enhancement on orbital MRI with contrast and the absence of any improvement in her visual field, which is typical of NAION, despite antiviral treatment [2]. The patient also had a history of hypertension and a disc-at-risk, which is a requirement for the development of NAION [3]. The optic disc edema resolved in the expected time course for NAION, and the patient had no other signs or symptoms of another optic neuropathy. There was also a clear temporal relationship between the IOP spike and the loss of vision. Although CSF was not obtained, this would be an extremely low-yield test, especially with no retrobulbar involvement seen on MRI.

NAION has been previously described to develop in response to elevated IOP and subsequent disruption to the optic nerve head perfusion pressure [4-6]. This has been well-described in a number of situations including after ophthalmological procedures, acute glaucoma, hypertensive uveitis, or the administration of steroid eye drops [7-11]. Cases of ophthalmological procedures include cataract extraction complicated by capsular block, pars plana vitrectomy for macular hole, and trabeculectomy complicated by NAION have been described [7-9]. NAION after intravitreal injections have also been presumed to be related to elevated intraocular pressure, although the IOP has not been routinely measured in most of these cases [12]. The presumed mechanism of NAION after cataract surgery has also been thought to be related to elevated IOP, which is elevated intraoperatively and sometimes postoperatively [13]. Acute primary angle closure and traumatic angle recession glaucoma has been reported to be complicated by NAION and may be bilateral and severe $[10,11]$. We were unable to retrieve any previously reported case of NAION secondary to herpes zoster anterior uveitis, but cases after Posner-Schlossman syndrome (PSS) have been described. Similar to our case, the PSS cases all had IOP in excess of $50 \mathrm{mmHg}$ and some cases were in individuals younger than 50 years of age $[5,10]$. We were unable to retrieve any cases of NAION that developed after steroid-related IOP spikes.

Elevated intraocular pressure is well-known complication of topical steroids and to a lesser extent systemic and topical steroid treatment $[14,15]$. This typically onsets around 36 weeks after the initiation of topical steroids and is thought to be related to increased resistance to aqueous outflow at the trabecular meshwork. The mechanism of this IOP elevation is unclear but the discontinuation of steroid treatment predictably triggers the reversal of IOP elevation back to baseline [14]. Complications of elevated IOP, not only include glaucoma, but may also result in retinal vein occlusions, corneal edema and as we have shown in this case, 


\section{Case Reports in Ophthalmology}

NAION. It is important that ophthalmologists closely monitor patients on topical steroids since NAION may be a rare and irreversible complication of their use. This may even occur in younger patients who may be taking topical steroids postoperatively for eye surgery or for ocular inflammatory disorders. Treatment of steroid-induced ocular hypertension typically involves discontinuation of the steroid and use of topical and systemic IOP-lowering medications. The elevation of IOP in our patient was likely related to topical steroid use since there was no active inflammation at the time of vision loss and the IOP responded promptly to the discontinuation of steroids.

In summary, the development of an optic neuropathy in close temporal relationship may not necessarily be related to VZV. We report a case of steroid-induced ocular hypertension for initial treatment of herpes zoster-related anterior uveitis that was complicated by NAION. Patients on topical steroids should be closely monitored since irreversible optic nerve damage may occur in this context.

\section{Statement of Ethics}

This study was carried out in accordance with the World Medical Association Declaration of Helsinki. Verbal and written consent were obtained from the patient.

\section{Conflict of Interest Statement}

The authors have no conflicts of interest to declare.

\section{Funding Sources}

No funding was received.

\section{Author Contributions}

Conception and design (P.H.Y., J.A.M.), data extraction (P.H.Y., J.A.M.), manuscript preparation (P.H.Y.), final approval (J.A.M.). All authors have seen and approved the final version of the manuscript for publication.

\section{References}

1 Kedar S, Jayagopal LN, Berger JR. Neurological and ophthalmological manifestations of varicella zoster virus. J Neuroophthalmol. 2019 Jun;39(2):220-31.

2 Hayreh SS. Ischemic optic neuropathy. Prog Retin Eye Res. 2009 Jan;28(1):34-62.

3 Tsai RK, Liu YT, Su MY. Risk factors of non-arteritic anterior ischemic optic neuropathy (NAION): ocular or systemic. Kaohsiung J Med Sci. 1998 Apr;14(4):221-5.

4 Slavin ML, Margulis M. Anterior ischemic optic neuropathy following acute angle-closure glaucoma. Arch Ophthalmol. 2001 Aug;119(8):1215.

5 Kim R, Van Stavern G, Juzych M. Nonarteritic anterior ischemic optic neuropathy associated with acute glaucoma secondary to Posner-Schlossman syndrome. Arch Ophthalmol. 2003 Jan;121(1):127-8.

6 Choudhari NS, George R, Kankaria V, Sunil GT. Anterior ischemic optic neuropathy precipitated by acute primary angle closure. Indian J Ophthalmol. 2010 Sep-Oct;58(5):437-40. 


\section{Case Reports in Ophthalmology}

\begin{tabular}{l|l}
\hline Case Rep Ophthalmol 2021;12:11-15 \\
\hline DOI: 10.1159/000511100 & $\begin{array}{l}\text { @ 2021 The Author(s). Published by S. Karger AG, Basel } \\
\text { www.karger.com/cop }\end{array}$ \\
\hline
\end{tabular}

Yuan and Micieli: NAION after HZO

7 Cunha LP, Cunha LV, Costa CF, Monteiro ML. Nonarteritic anterior ischemic optic neuropathy following pars plana vitrectomy for macular hole treatment: case report. Arq Bras Oftalmol. 2016 Sep-Oct;79(5):342-5.

8 Lee H, Kim CY, Seong GJ, Ma KT. A case of decreased visual field after uneventful cataract surgery: nonarteritic anterior ischemic optic neuropathy. Korean J Ophthalmol. 2010 Feb;24(1):57-61.

9 Kawashima H, Nagai N, Shinoda H, Tsubota K, Ozawa Y. Optic neuropathy causing vertical unilateral hemianopsia after pars plana vitrectomy for a macular hole: A case report. Medicine (Baltimore). 2018 Apr;97(17):e0321.

10 Irak I, Katz BJ, Zabriskie NA, Zimmerman PL. Posner-Schlossman syndrome and nonarteritic anterior ischemic optic neuropathy. J Neuroophthalmol. 2003 Dec;23(4):264-7.

11 Nahum Y, Newman H, Kurtz S, Rachmiel R. Nonarteritic anterior ischemic optic neuropathy in a patient with primary acute angle-closure glaucoma. Can J Ophthalmol. 2008 Dec;43(6):723-4.

12 Battaglia Parodi M, Iacono P, Cascavilla ML, Zucchiatti I, Kontadakis DS, Vergallo S, et al. Sequential anterior ischemic optic neuropathy and central retinal artery and vein occlusion after ranibizumab for diabetic macular edema. Eur J Ophthalmol. 2010 Nov-Dec;20(6):1076-8.

13 Yang HK, Park SJ, Byun SJ, Park KH, Hwang JM. Risk of Nonarteritic Anterior Ischemic Optic Neuropathy After Cataract Surgery. Am J Ophthalmol. 2019 Nov;207:343-50.

14 Becker B, Mills DW. Elevated intraocular pressure following corticosteroid eye drops. JAMA. 1963 Sep;185(11):884-6.

15 Bernstein HN, Mills DW, Becker B. Steroid-induced elevation of intraocular pressure. Arch Ophthalmol. 1963 Jul;70(1):15-8.

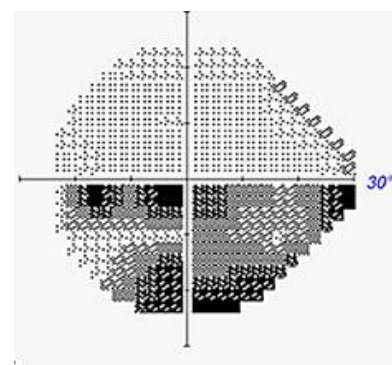

Pattern Deviation

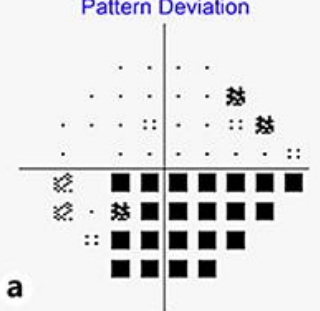

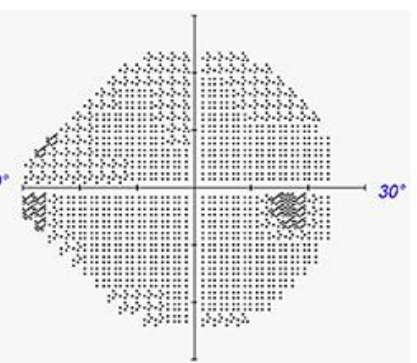

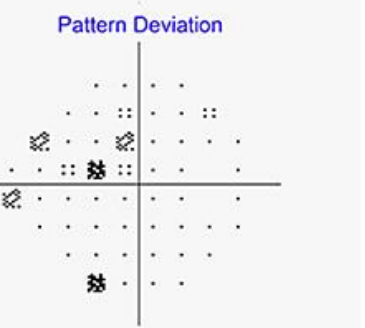

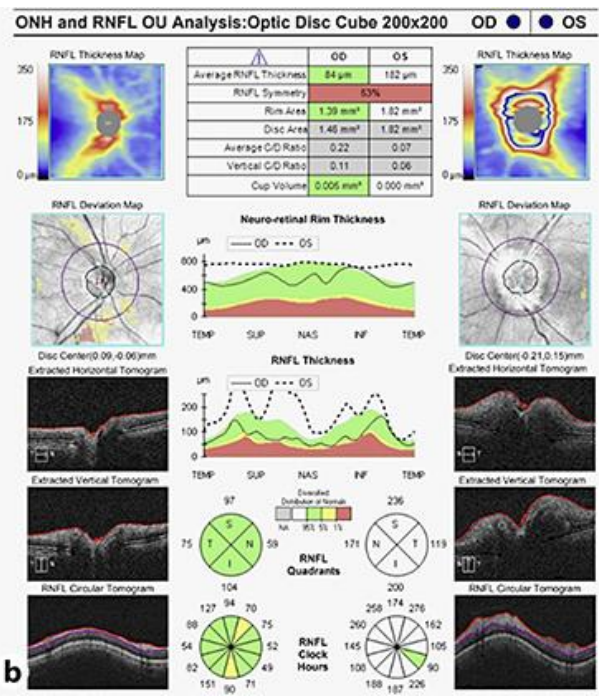

Fig. 1. a Humphrey 24-2 SITA-Fast visual fields demonstrating a left inferior altitudinal visual field defect. b Optical coherence tomography of the retinal nerve fiber layer (RNFL) at presentation with left eye vision loss demonstrating an elevated RNFL due to optic disc edema in the left eye. 